\title{
Persistent impairment of taste associated with terbinafine
}

\author{
A. J. Duxbury, ${ }^{1}$ R. J. Oliver, ${ }^{2}$ and M. N. Pemberton, ${ }^{3}$
}

\section{A second case of persistent taste disturbance associated with terbinafine is described. Taste disturbance associated with this drug is reviewed and a table is provided listing the more common drugs associated with taste disturbance.}

\begin{abstract}
$\mathrm{T}$ erbinafine is an antifungal drug first introduced in the UK in 1991. It is widely used topically and systemically in the treatment of dermatophyte infections including fungal infections of the nails where treatment for 6 to 12 weeks or longer may be necessary to effect a cure. In general terbinafine is well tolerated, but its side effects include a disturbance of taste that in a large surveillance study was found to be experienced by some $0.6 \%$ of patients. ${ }^{1}$ In this study taste disturbance was reported as being only transient with full recovery occurring on stopping the drug. There has, however, been one report in the literature of persistent taste impairment. ${ }^{2}$ In this case partial recovery occurred on stopping terbinafine but there was still incomplete recovery 3 years later. We wish to report a further case of persistent taste disturbance associated with terbinafine.
\end{abstract}

\section{Case report}

A 51-year-old woman was referred to the Unit of Oral Medicine by her general dental practitioner for the investigation of an altered taste sensation and recurrent ulceration affecting the lateral borders of her tongue. The patient reported an altered taste and the inability to taste sweet foods, including sugar. Symptoms had arisen 18 months prior to referral during a course of terbinafine prescribed for a toenail infection. Initially the patient experienced a

${ }^{1}$ Senior lecturer in Dental Pharmacology and Therapeutics and Oral Medicine, ${ }^{2}$ Lecturer in Oral Medicine and Oral Surgery, ${ }^{3}$ Lecturer in Oral Medicine, University Dental Hospital of Manchester, Higher Cambridge Street, Manchester, M15 6FH Correspondence to: A. J. Duxbury

REFEREED PAPER

Received 12.08.99; accepted 28.10.99

(c) British Dental Journal 2000; 188: 295-296 generalised loss of taste and this was followed by a persistent bitter taste. Terbinafine was stopped some 2 months into a 12-month course of therapy in consultation with her medical practitioner. During the past year her taste sensation had partially recovered. Eating, drinking and tooth brushing however still triggered the current symptoms of a bitter aftertaste accompanied by increased salivation. On eating, the first few mouthfuls tasted relatively normal before a bitter taste predominated. Prior to her loss of taste the patient had had an upper respiratory tract infection to which she ascribed the initial taste loss. Failure to recover taste subsequently led to stopping terbinafine.

Medically the patient had been taking medication for tachycardia (propranolol $10 \mathrm{mg}$ ) and acne rosacea (tetracycline $250 \mathrm{mg}$ ) on a regular basis for several years including the period when she took terbinafine. She gave a history of allergies to penicillin, trimethoprim and erythromycin. She had no history of neurological or nasal problems apart from a

\section{In brief}

- Disorders of taste and smell are not uncommon

- Medication is often implicated with more than 200 listed drugs. Withdrawal of the offending agent usually results in alleviation of the problem but it may persist

- Loss or disturbance of taste is socially disturbing, can make patients distressed, and can lead patients to seek medical and dental advice

- Once simple medical and dental causes have been excluded investigation by specialist departments is appropriate minor sinus operation as a child. She did not smoke and drank alcohol only occasionally. On examination the oral soft tissues and dental hard tissues were all clinically healthy and no abnormalities were detected. The ulceration noted by her general dental practitioner had resolved and did not recur during the follow-up period. The results of a full blood count, random blood glucose, serum urea and electrolytes and liver function tests, were within normal ranges.

\section{Discussion}

Overall $80 \%$ of all taste and smell disorders are said to have an identifiable cause while the rest are considered idiopathic with no discernable aetiology. ${ }^{3}$ There are close to 200 conditions that can lead to chemosensory disorders and these have been covered in reviews. ${ }^{3}$ Medication is a common cause of taste disorders with more than 200 drugs listed. A list of some of the more important medications is given in Table $1.3,4$ Terbinafine (LAMISIL $^{\mathrm{R}}$ ) is known to have several side-effects, the most commonly reported of which include headache, diarrhoea, dyspepsia and abdominal pain, ${ }^{5}$ other reported adverse reactions include taste loss. A post market surveillance study of terbinafine involving 10,000 patients in 1996 reported that all patients with taste loss fully recovered this sensation and there was no evidence to suggest the effect was irreversible. $^{1}$ Recovery times varied between 2-186 days (average 6 weeks). Recovery was usually gradual but could be abrupt. A review by Novartis Pharmaceuticals UK Ltd (personal communication) of reported cases of taste loss found some 5\% of patients had had loss of taste for 12 weeks or longer.

Persistent loss of taste associated with terbinafine would however appear to be extremely rare. The only other recorded case of persistant taste loss we could find was a patient who developed an inability to taste coffee or tea and required more salt in food some 6 weeks after commencing terbinafine. ${ }^{2}$ Shortly afterwards complete taste loss arose and terbinafine was 


\section{Table 1 Drugs offecting teste and smell 5,8}

\section{Class of medication}

stopped immediately. Recovery was partial with continued difficulty tasting sugar or salt in food some 3 years later. In comparison our patient described partial loss of sweetness with a persistent bitter taste, followed by a partial recovery over an 18 month period.

The taste disturbance associated with terbinafine is thought to be associated with taste receptor dysfunction through the inhibition of cytochrome P-450 dependent enzymes, although the exact mechanism is unclear. ${ }^{2,6}$ Predisposing factors have been reported as being a low body mass index and advanced age, ${ }^{7}$ while some patients have been found to have a reduced intake of protein and zinc. A higher tissue concentration of terbinafine may occur in slim people and a reduction in taste buds with increasing age may exacerbate the problem. Zinc has been reported to have a role in the normal appreciation of taste and smell and zinc salts have been used in the treatment of disordered function of these modalities, but other reports claim this has not been convincingly shown. ${ }^{3}$ The ability to taste bitter and salty food is considered to be lost more often than that of sweet and sour tastes because of the lower number of receptors related to the former two taste modalities. $^{2}$

Links between these factors and our own patient exist. Our patient was middleaged and prior to exposure to terbinafine she was under weight. At the time she took terbinafine her weight was 7.5 stones (47.7 kgs) while currently her weight at 9.0 stones $(57.3 \mathrm{kgs})$ is normal for her height. However she ate a varied diet, there were no chronic nasal or sinus problems, and she was taking no over-the-counter drugs. Her medication then and now, was of propranolol and tetracycline. Propranolol is not, as far as we are aware, implicated in taste disturbance. Tetracycline may cause stomatitis and has been reported previously, as have other antibiotics, as a cause of taste disturbance (Table 1). Our patient took tetracycline prior to the development of her taste disturbance without any effect on taste; the involvement of this drug therefore seems unlikely. The increased

Anti-inflammatories
Antidiabetics

Anticoagulants

Antihistamines

Antimicrobials

\section{Drug (side effect)}

Glipizide. Biguanides (metallic taste)

Phenindione (dysgeusia)

Chlorpheniramine

Amphotericin (dysgeusia), ampicillin, cephamandole, griseofulvin (dysgeusia), lincomycin, levamisole, metronidazole (metallic taste), sulphasalazine, streptomycin, tetracyclines, terbinafine, tyrothrycin

Antineoplastics and immunosuppressants

Bleomycin (sour and metallic taste and taste loss), doxorubicin, methotrexate (sweet taste), azothiaprine, carmustine, vincristine.fluorouracil (altered bitter and sour taste)

Antirheumatics

Antiseptics

Antithyroid

Antihypertensives and diuretics

Anti-arrhythmics

Antipsychotics and anticonvulsants

Local anaesthetics

Muscle relaxants, antiparkinson agents

Lipid modulating agents

Opiates

Sympathomimetics

Salicylates (taste disturbance) phenylbutazone (hypogeusia, ageusia)

Allopurinol (metallic taste), colchicines, gold (metallic taste), penicillamine (reversible loss of taste)

Chlorhexidine (aftertaste), hexetidine, sodium lauryl sulphate (loss of sweet and salt taste)

Carbimazole

Captopril (and other ACE inhibitors), diazoxide, ethacrynic acid

Propafenone, amiodarone

Carbamazepine (hypogeusia, ageusia), lithium (dysgeusia), phenytoin, trifluoperazine, zopiclone (bitter or metallic taste)

Benzocaine etc.

Baclofen (ageusia, hypogeusia), levodopa (taste disturbance)

Clofibrate

Codeine, morphine

Amphetamines salivary flow claimed by the patient may be postulated as arising in response to the sour/bitter taste. ${ }^{8}$

In this case, the temporal relationship between the onset of symptoms and the prescribing of terbinafine, and the known side effects of terbinafine make it likely that the two events are causally related. The role of a viral infection at the time the loss of taste arose, or an interactive effect with tetracycline, however, cannot be totally discounted. We report this case to draw attention to the rare occurrence of persistent taste loss associated with terbinafine and the overall problem of taste disturbance associated with a variety of drugs that may be taken by patients attending the dental surgery (Table 1). Loss or disturbance of taste is socially disabling, can make patients depressed, and leads them to seek medical and dental advice. Once simple medical and dental causes have been excluded further investigation by specialist departments is appropriate.
O'Sullivan D P, Needham C A, Bangs A, Atkin K, Kendall F D. Post marketing surveillance of oral Terbinafine in the UK: report of a large cohort study. Br J Clin Pharmacol 1996; 42: 559-565.

2 Bong J L, Lucke T W, Evans C D. Persistent impairment of taste resulting from terbinafine. Br J Dermatol 1998; 139:747-748.

3 Lamey P J. Salivary Gland and Chemosensory Disorders. In Millard H D and Mason D K, (eds) Perspectives on 2nd World Workshop on Oral Medicine. Section 4. pp 273-360. Chicago: University of Michigan, 1993.

4 Duxbury A J. Systemic Pharmacotherapy. In Jones J H and Mason D K, (eds) Oral Manifestations of Systemic Disease. Chapter 11. pp 411-479. London: Balliere Tindall 1990.

5 British National Formulary. No.38 p280. London: British Medical Association and the Royal Pharmaceutical Society of Great Britain, 1999.

6 Henkin R I. Drug induced taste and smell disorders incidence, mechanisms and management related primarily to treatment of sensory receptor dysfunction. Drug Safety 1994; 11: 318-377.

7 Stricker B H, Van Riemsdijk M M, Sturkenboom M C, Ottervanger J P. Taste loss to Terbinafine: a case - control study of potential risk factors. Br J Clin Pharmacol 1996; 42: 313-318.

8 Spielman A I. Interaction of saliva and taste. $J$ Dent Res 1990; 69: 838-843. 